\title{
The Epoch of Reionization: Observing Galaxies in Their Cradle
}

\author{
Kasper B. Schmidt* \\ Department of Physics, University of California, Santa Barbara, CA, 93106-9530, USA, and \\ Leibniz-Institut für Astrophysik Potsdam (AIP), An der Sternwarte 16, 14482 Potsdam, Germany \\ E-mail: kbschmidteaip.de
}

The epoch of reionization at the infancy of the Universe is one of the observational frontiers of modern astronomy. It has been the key focus of numerous studies over the past decade. Through an impressive advancement of observational capabilities both on the ground and in space, these efforts have resulted in a tremendous gain of insight. Even though many questions about the early Universe are still unanswered, with more than a thousand galaxies known at redshifts greater than 6 , we understand the formation and life of the first galaxies better than ever before. I will present an observer's view of the galaxies at the epoch of reionization, describing the preferred tool used to study the high-redshift Universe, the rest-frame UV luminosity function, and the main insights this has let to in recent years. The spectroscopic follow-up of photometrically pre-selected highredshift galaxy samples has an equally great predictive power about the epoch of reionization. I will illustrate this by presenting recent studies inferring the content of ionized hydrogen in the early Universe from spectroscopic follow-up campaigns.

BASH 2015

18 - 20 October, 2015

The University of Texas at Austin, USA

${ }^{*}$ Speaker. 


\section{Introduction: The Epoch of Reionization}

With the last few decades' advancement of near-infrared (NIR) detectors and NIR spectrographs at the largest facilities on the ground including ESO's Very Large Telescope (VLT) and the Keck telescopes, and in particular with the last service mission to the Hubble Space Telescope (HST) and the installment of the Wide Field Camera 3 (WFC3) in 2009, it has become possible to observe and take spectra of galaxies at redshifts beyond 6. At these redshifts the Universe was undergoing a phase transition from an intergalactic medium (IGM) completely filled with neutral hydrogen to a mostly ionized IGM. After the ionized 'plasma' existing immediately after the Big Bang recombined at the last scattering surface at $z \sim 1100$, this second phase transition is referred to as the Epoch of Reionization (EoR) and roughly took place when the Universe was between 200 Myr and 900 Myr old. Figure 1 illustrates the evolution of the Universe from the last scattering surface to the present epoch where the IGM is fully ionized, marking the location of the EoR in both redshift and time.

The Epoch of Reionization (EoR):

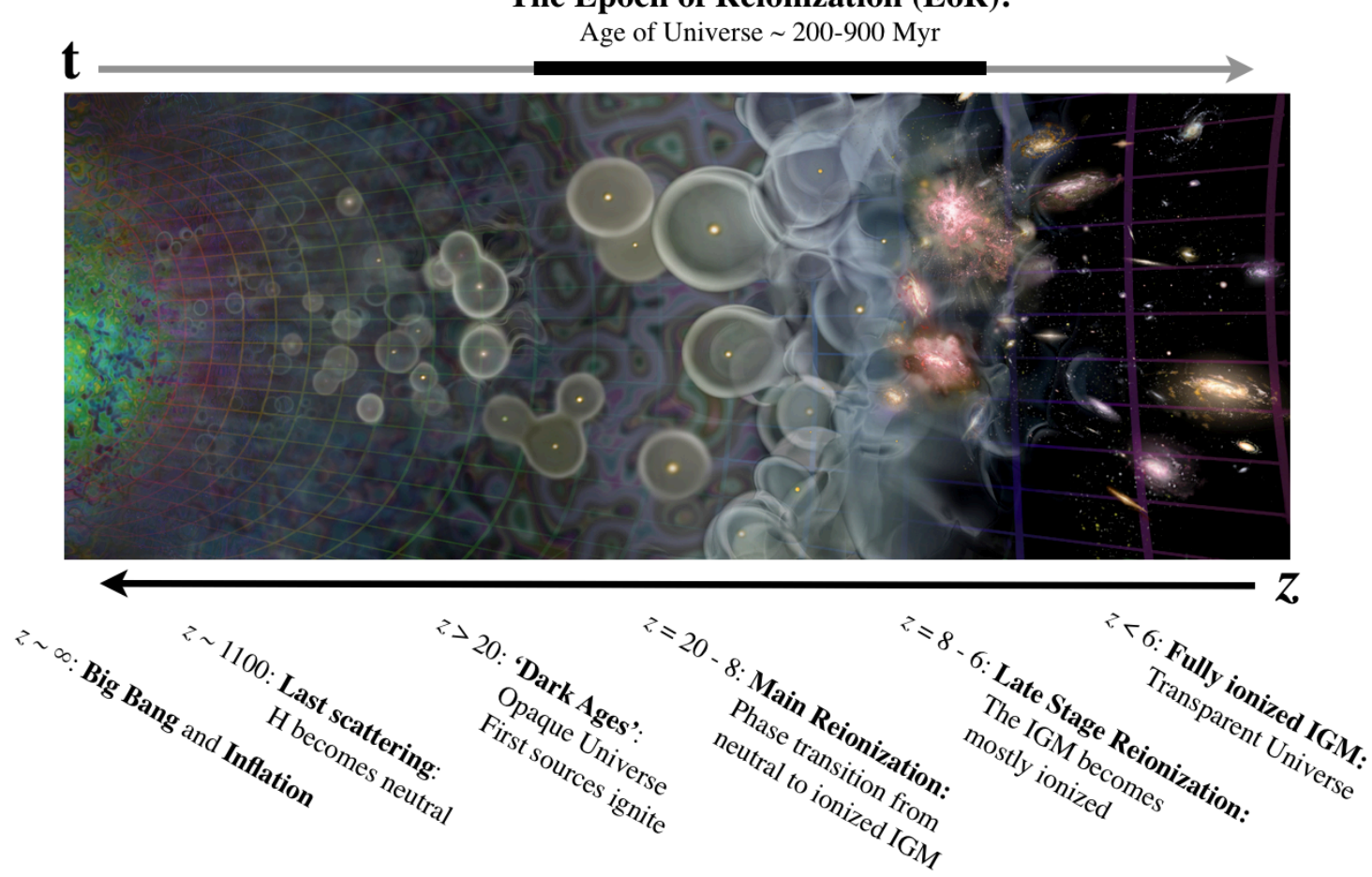

Figure 1: Artist impression of the evolution of the Universe adapted from [1]. The main epochs of the early Universe have been marked on the redshift axis below the illustration. The EoR indicated on the time axis above the illustration marks the phase transition from a fully neutral intergalactic medium to an ionized intergalactic medium at $z \sim 6-20$. The EoR is the current observational frontier for galaxies and provides vital information about the early Universe and the early stages of galaxy formation and evolution.

Currently the EoR is the observational frontier for galaxies, and has proven extremely valuable in mapping the early evolutionary stages of galaxy formation and galaxy evolution when the Universe was only a few per cent of its current age. Even though the early Universe has been 
studied in great detail in recent years, which the list of references on page 15 is a testimony to (see also references in, e.g., [2, 3, 4, 5]), there are still many questions about the EoR and the galaxies that inhabit this epoch, which are heavily debated in the literature. These include the discussion of which objects actually reionized the Universe. There have been claims that active galactic nuclei $[6,7]$ provide a significant amount of reionizing photons. However, the current consensus in the high-redshift community points towards (faint) galaxies being the main contributor of the ionizing radiation being responsible for ionizing the neutral hydrogen in the IGM [8, 9, 10, 11]. Having decided where the contribution to the reionization comes from, an important aspect of describing the EoR and assessing the effects it has on galaxy formation and evolution, is to estimate how much radiation these ionizers produce, and if this radiation is enough to sustain an ionized IGM, as a sudden burst of ionizing radiation would only ionize the IGM for a short while before it recombines. Such estimates enable studies of the duration of the EoR and how this affects the continued galaxy formation. The samples of galaxies at the EoR themselves have also been studied and debated in great detail. In particular, comparisons with $z<5$ galaxy reference samples have proven very valuable and have provided insights into the size [12, 13, 14], luminosity [15, 16], and star formation $[17,18]$ evolution of galaxies over the vast majority of the life of the Universe.

In this proceeding, I will highlight some of these findings and give a broad overview of the current state of affairs seen through the eyes of a NIR observer. I will describe the observations and the photometric selection techniques used to find the galaxies at the EoR in Section 2. In Section 3, I will describe the favorite tool of the EoR observer, the rest-frame UV luminosity function, which is being used to analyze the obtained galaxy samples and to infer information about both the EoR and the galaxies themselves. In Section 4 and 5, I will focus on the outcomes of some of the more recent studies of the luminosity functions at the EoR and of NIR ground- and space-based spectroscopy of galaxies at $z>6$. I will finish with a summary in Section 6 before a short outlook highlights the bright and very promising future of EoR studies in Section 7.

\section{Observing the Early Universe}

Observing and detecting galaxies in the early Universe at redshifts above 6 heavily relies on the shape of the observed galaxy spectral energy distribution (SED). As neutral hydrogen absorbs photons at $1216 \AA$ (and re-emits them in an arbitrary direction, i.e. scatters them) the optical depth of photons at rest-frame $1216 \AA$ is strongly dependent on the amount of neutral hydrogen in the medium they are being emitted into. A fully neutral IGM will therefore be opaque to Ly $\alpha$ emission which is emitted at $1216 \AA$. As the Universe is expanding, photons will be redshifted to higher wavelengths (lower energies), meaning that a photon emitted at $1150 \AA$ will be redshifted to $1216 \AA$ after traveling a finite amount of time through the IGM, and be obscured by intervening neutral hydrogen. Therefore, a fully neutral medium can potentially absorb all photons emitted short-wards of rest-frame $1216 \AA$. This effect, resulting from the increasingly neutral IGM at higher redshifts, is clearly seen in imaging $[19,20,21]$ and spectra $[22,23,24]$ of high redshift galaxies as illustrated in Figure 2. The two panels in Figure 2 show a small sample of template galaxy SEDs from $z=3$ to $z=9$. The left panel shows a zoom-in of the observed NIR wavelengths, where the decreasing flux blue-wards of the rest-frame $1216 \AA$ is seen. For reference, the modeled broad band magnitudes 
for a sub-sample of $H S T$-filters are over plotted the galaxy SEDs. This characteristic decrease of rest-frame UV radiation is referred to as the Lyman break.

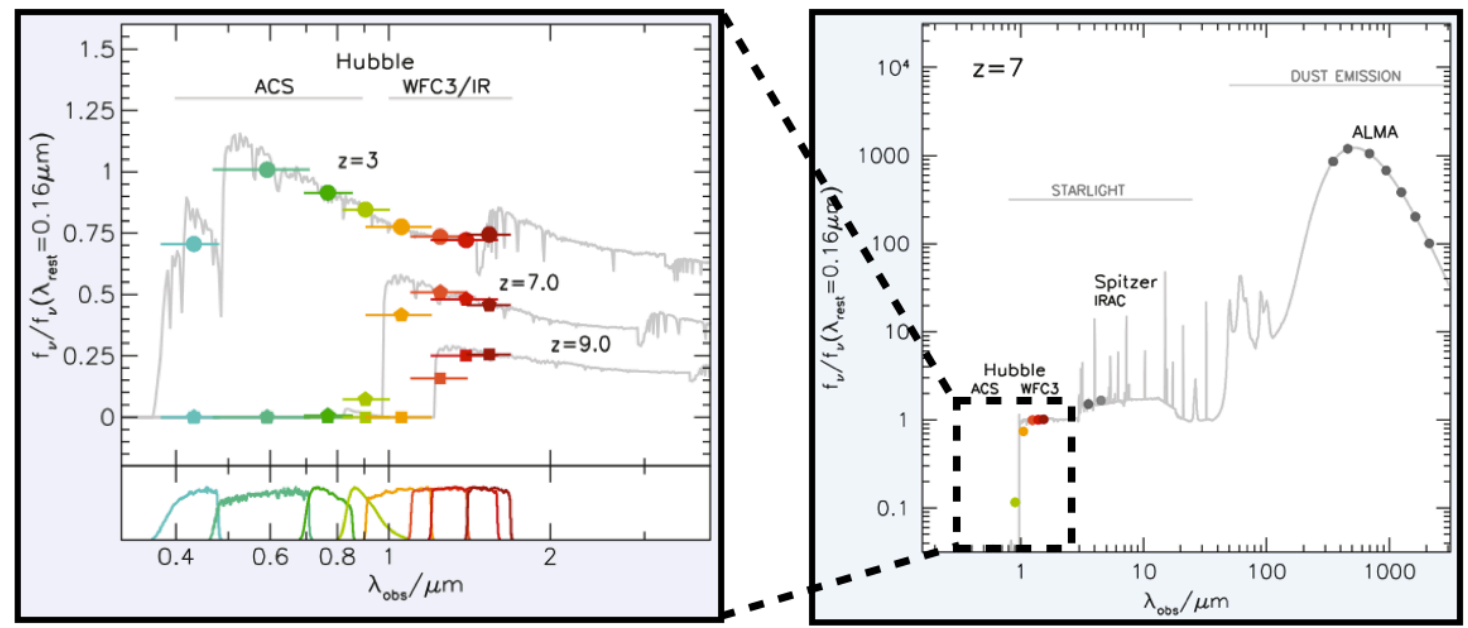

Figure 2: Examples of template galaxy SEDs in the observed frame for star forming galaxies, illustrating the lack of radiation blue-wards of the Ly $\alpha$ line at rest-frame $1216 \AA$, known as the Lyman break. The left panel shows the region around the Lyman break for a small sample of galaxy templates at redshifts 3-9. At high redshift the Lyman break moves into the NIR, such that these galaxies can be selected as 'dropouts' in the bluest HST filters shown in the left panel. The mid- and far-infrared wavelengths (right panel) are dominated by starlight and dust emission. Figure adapted from [25].

The so-called Lyman break selection technique was pioneered at low and intermediate redshifts $[26,27,28]$. It exploits the Lyman break by applying cuts on the broad band colors and the detection thresholds in multi-band photometry. The general requirements are: 1) that the object is not detected in the photometric bands blue-wards of the break (rest-frame far-UV), 2) that the two bands straddling the break show a pronounced red color, as the majority of the flux will be in the reddest band, and 3) that there is a clear detection in the band(s) red-wards of the break, as illustrated in the left panel of Figure 2. At redshift 7 the Lyman break occurs in the observed NIR, which has previously complicated the selections due to the limitations of NIR detectors. A galaxy selected with the Lyman break technique is referred to as a Lyman break galaxy (LBG) or a dropout galaxy, as it 'drops out' of bands short-ward of the Lyman break.

As the selection of LBGs is solely based on photometry, the selected sources are only candidate high-redshift galaxies, and samples of LBGs are known to be contaminated. Contaminants in this context are objects at lower redshift mimicking the colors of the LBGs resulting in falsepositives in the color selections. Both brown dwarfs and low-redshift galaxies are known to contaminate LBG samples [3, 29, 30, 31, 32]. This is a consequence of both pronounced spectral breaks in these sources' SEDs and lack of depth in the photometry short wards of the break. Estimated contamination rates of LBG samples range from a few percent with good photometric coverage and depth to over $40 \%$ for minimal photometry [16, 21, 33, 34].

A second photometric selection technique widely used to find high-redshift galaxy candidates is the photometric redshift technique. The photometric redshift technique is closely related to the 
Lyman break technique, as it uses the characteristic Lyman break in the SED to obtain a photometric redshift based on fits to SED templates from stellar population synthesis models similar to the ones shown in Figure 2. There is a wide range of models and fitting codes available (see, e.g., the lists at http://sedfitting.org/) and they have proven very useful in the identification of high-redshift galaxies $\left[15,35,36,37{ }^{1}\right.$

The Lyman break selection and the photometric redshift selection, are currently the two main selection techniques for obtaining large samples of high-redshift galaxies and have been shown to produce consistent results [15, 35, 39, 40]. However, a growing list of alternative selection techniques are currently being studied and applied to data to search for galaxies in the early Universe. These include selections based on an excess in Spitzer Space Telescope photometry due to restframe optical emission lines [41, 42, 43], narrow-band searches for Lyman alpha emitters (LAEs) looking for flux-excess between a narrow and a broad band indicative of the $\operatorname{Ly} \alpha$ emission line $[2,44,45,46]$, and most recently, searches using rest-frame UV lines unaffected by the increasing amount of neutral hydrogen in the IGM at high redshift like CIII] and CIV have been initiated $[47,48]$. These selections have all contributed to the growing number of high-redshift candidate galaxies available today. However, with the improvement of the available NIR instruments over the last decade, especially the Lyman break selection technique and photometric redshift technique has revealed extensive high-redshift galaxy samples, such that today thousands of galaxy candidates are known at the EoR at $z>6[16,32,49]$. These galaxy samples are providing an unprecedented possibility to explore the physics governing the EoR and the early Universe.

\section{The Favorite Tool of the High- $z$ Observer: The UV Luminosity Function}

The main tool used to analyze the available high redshift galaxy samples is the luminosity function (LF). The LF essentially counts the number of galaxies at a given luminosity describing the population as a whole at the redshift the galaxy sample defines. The Schechter function [50] is often used to parameterize the LF, and gives a simple relationship between the empirical power-law behavior of galaxy samples at the faint end, and the seemingly exponential decline of the number of very bright galaxies. In-between these two regimes a smoothly varying transition at intermediate luminosities, often referred to as the 'knee' of the luminosity function, mark the characteristic galaxy population luminosity, referred to as $L^{\star}$. Hence, an $L^{\star}$ galaxy is an above-average bright galaxy describing its parent population. A sub- $L^{\star}$ galaxy of, e.g., $0.1 \times L^{\star}$ is considered a dwarf galaxy for the given galaxy sample. The left panel of Figure 3 shows a schematic of the standard Schechter LF, marking the characteristics of the Schechter equation given in the figure. The right panel shows an actual UV LF at redshift 8 obtained from $H S T$ data from the BoRG [21, 51, 52] and HUDF [49] surveys. Recently, the intrinsic shape of the LF has been discussed, as there have been indications of a non-exponential decline at the bright end at high redshift [15, 53, 54, 55], and because the bright end of the LF is known to be affected by the so-called magnification bias $[10,56,57,58,59]$.

\footnotetext{
${ }^{1}$ SED fitting is not only useful for obtaining photometric redshifts of high redshift galaxies. SED fitting is an industry in its own right, with the possibility of determining much more than just the redshift of galaxies, including the stellar masses, star formation rates, star formation histories, dust attenuation, dust emission, etc. A detailed description of SED fitting is beyond the scope of this proceeding but see, e.g., [38] and references therein.
} 

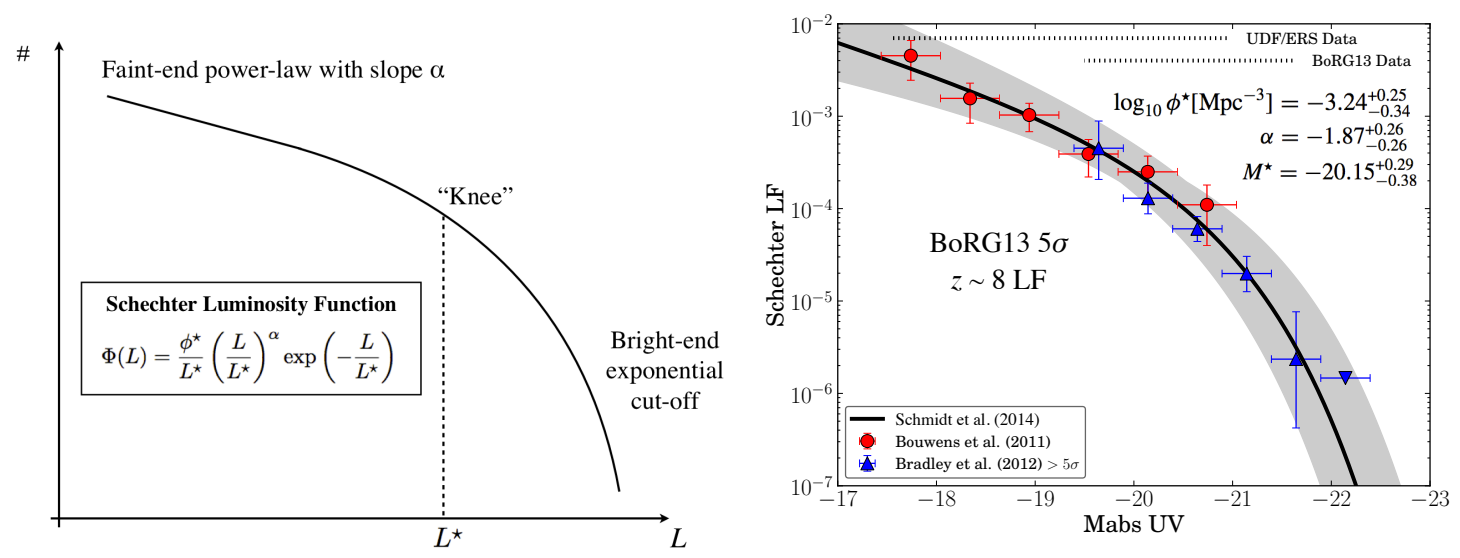

Figure 3: A schematic cartoon of the standard Schechter LF (left panel), marking the faint-end power-law behavior, the 'knee' of the LF, and the exponential cut-off at the bright end characteristic of the Schechter expression (inserted equation). The $\phi^{\star}$ determines the overall normalization of the LF and $L^{\star}$ is the characteristic luminosity at the 'knee'. The right panel shows the redshift 8 UV LF from the HUDF+BoRG LBGs [21]. The LF is fitted with a Bayesian framework avoiding binning of the data. The binned LF (points with error bars $[49,52])$ is shown for reference.

When focusing on galaxies at the EoR, the most informative LF is determined at the restframe UV. The rest-frame UV LF directly relates to the number of ionizing photons provided by the galaxy population it describes. In particular, the LF estimates the total number of galaxies represented by the observed sample of galaxies, as $\int_{L \text { limit }} L F d L=N_{\text {total }}\left(L>L_{\text {limit }}\right)$, as well as the radiation these provide through the UV luminosity density $\rho_{L}=\int L \times L F d L$. Furthermore, the UV LF is a proxy for the star formation rate density, as it has been shown empirically that there is a direct relation between the UV luminosity and the star formation rate [60, 61], namely, $L_{\mathrm{UV}}=$ $\left(\mathrm{SFR} /\left[M_{\odot} \mathrm{yr}^{-1}\right]\right) 8.0 \times 10^{27} \mathrm{erg} \mathrm{s}^{-1} \mathrm{~Hz}^{-1}$. By assuming that the Schechter profile extends below the luminosity observation limit, these quantities can be estimated for faint unobservable galaxies, which are also contributing to the overall ionizing photon budget. As a note of caution, to get sensible limits from (any) extrapolation you need to know your functional form with high precision. For the LF it is the precision of the faint-end power-law slope $\alpha$, which is the constraining factor. And as $L^{\star}$ and $\alpha$ are de-generate, it is crucial to get large samples at both the bright $\left(L>L^{\star}\right)$ and faint $\left(L<L^{\star}\right)$ end of the LF.

\section{The Luminosity Functions at the EoR}

Based on the available samples of galaxies at all redshifts, the 'community consensus' LFs are slowly being established further and further into the EoR. As recently summarized by [32], LFs have been presented all the way out to redshift 10. With such a large range of well-determined UV LFs, the evolution in the Schechter LF paramters become evident. As shown in Figure 4 the current consensus is that the galaxy LF does not have any significant evolution in $L^{\star}$ (or $M^{\star}$, if $L^{\star}$ is converted to absolute UV magnitude at the given redshift). On the other hand, as is clear from the central and right panels of Figure 4, there is a well-established evolution in both $\alpha$ and 
the overall normalization of the LF, $\phi^{\star}$. The faint-end slope becomes steeper at higher redshifts indicating a fractional larger contribution to the photon budget from faint dwarf galaxies at high redshift compared to low and intermediate redshifts. For current discussions of the evolution of the Schechter luminosity function parameters see, e.g., [15, 16, 32].
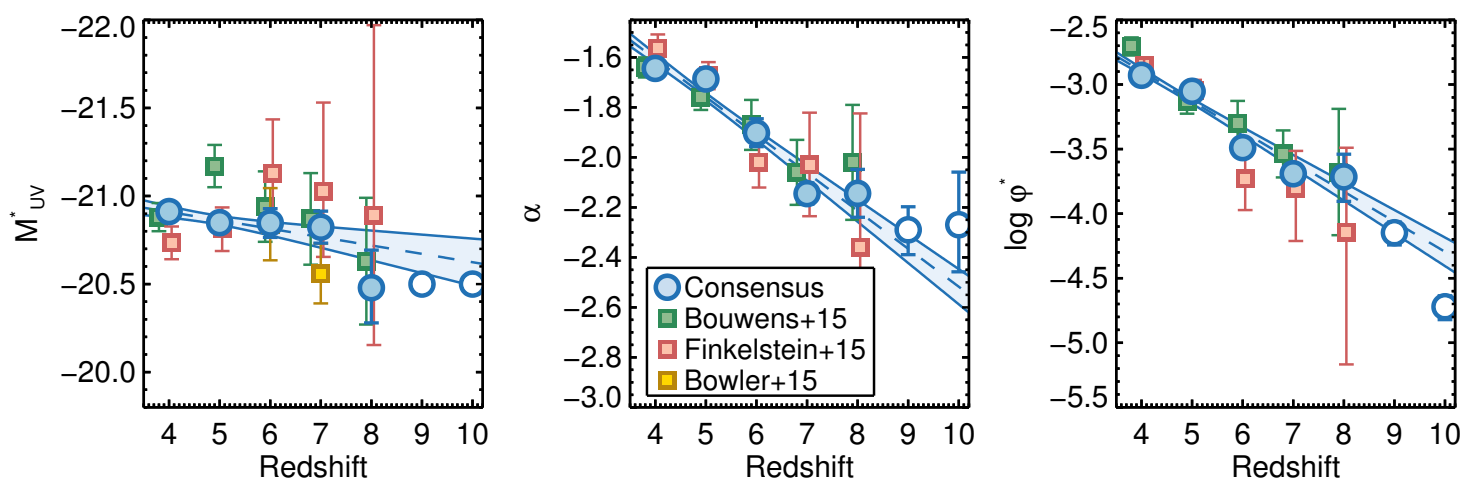

Figure 4: The evolution of the Schechter LF parameters from [32]. From left to right the evolution in $M_{\mathrm{UV}}^{\star}$ ( $L^{\star}$, the position of the LF 'knee'), the faint-end slope, $\alpha$, and the overall LF normalization, $\phi^{\star}$ are shown. Only $\alpha$ and $\phi^{\star}$ show pronounced evolution. The data points [15, 16, 53] summarize the current samples of LBGs at high redshift. When fitting the LF, the $M_{\mathrm{UV}}^{\star}$ has been fixed at $z=9$ and 10 (open blue circles) due to limited sample sizes at these redshifts.

The improvement in the UV LFs at the EoR has also greatly improved our understanding of the evolution of the cosmic star formation rate density (SFRD). Converting the luminosity density obtained from integrating the LFs at different redshifts down to a given absolute magnitude limit as described in Section 3, and exploiting the empirical conversion between the UV luminosity and the star formation rate, it has become clear that the SFRD continues to drop at the EoR, following the trend already seen at redshift 4 , after the star formation peaked at $z \sim 2-3$ [62]. This decline is illustrated in the cosmic SFRD shown in the left panel of Figure 5 [32]. With the advancement of $z>8 \mathrm{LFs}$, a steepening of the SFRD at $z>8$ compared to the extrapolation of the established relation (indicated by the dashed lines in Figure 5) has been observed [17, 63]. This affects the detectability of high redshift sources as star formation is needed to produce the observable radiation. The decline is expected from models of the SFRD, which is illustrated by the SFRD model shown in the right panel of Figure 5 [57]. This model links the star formation across cosmic time to the assembly of dark matter halos assuming a mass-dependent, but redshift-independent, efficiency, introducing a new self-consistent treatment of the halo star formation history. As can be seen, this simple model agrees very well with the current data. When this is combined with the fact that the sample sizes at $z>8$ are currently still very small, and that when estimating the SFRDs, they are estimated above a fixed absolute magnitude at all redshifts, as the integration limit of the LF is independent of redshift, there is currently no violation of the theoretical expectations by the data.

Another main focus of studies of galaxies at the EoR has been to infer the state of the IGM reionization. The fraction of ionized hydrogen depends on the balance between ionizing photons and the recombination time of hydrogen $[9,66,67]$. The density of ionizing photons can be related to the measured $\rho_{L}$ assuming a photon production efficiency, $\xi$, an escape fraction of the ionizing 

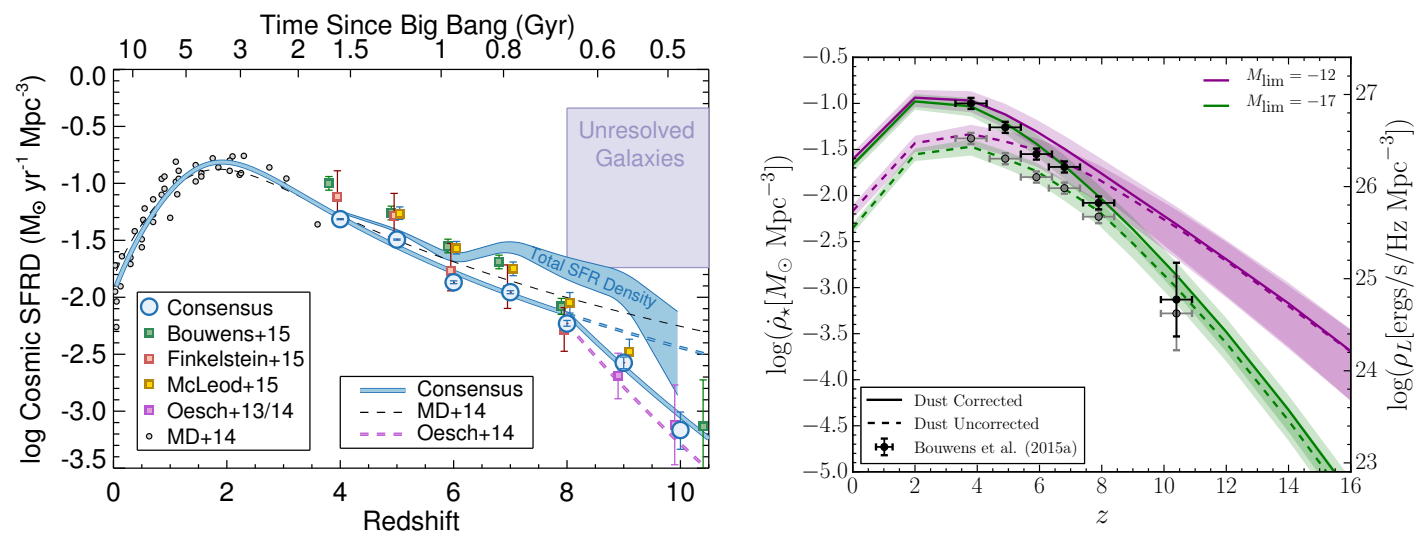

Figure 5: The star-formation rate density (SFRD) evolution over cosmic time taken from [32] (left panel). The low- $z$ data [62] and the continuously growing sample sizes of high redshift galaxies $[15,16,63,64,65]$ have shown that the SFRD is continuously growing until the peak of star formation at redshift 2-3 [62]. A potential change in the rate of growth at $z \sim 8$ is seen in current data (though sample sizes are limited at $z>8$ ). This is in good agreement with recent models as indicated by the SFRD models shown in the right panel [57]. This model links the star formation across cosmic time to the assembly of dark matter halos assuming a mass-dependent redshift-independent efficiency, and agrees well with current data [16].

photons, $f$ esc, and a so-called clumping factor of the IGM, $C$. These quantities are fairly uncertain and are usually assigned fiducial values based on both empirical estimates from data and theoretical models $[67,68,69,70,71,72,73]$. Assuming values for the escape fraction, clumping factor, and the ionizing photon production efficiency, the UV LF can be used to infer the ionized fraction of the IGM, $Q$. In particular, the BoRG $z \sim 8$ LF [21] shown in the right panel of Figure 3 predicts $Q$ as a function of LF integration limit as shown in the left panel of Figure 6. This inference shows that, based on the BoRG galaxy sample, a fully ionized Universe at $z \sim 8$ is only likely if dwarf galaxies as faint as $M_{\mathrm{UV}} \sim-15$ are included in the photon budget. And that is only for the most optimistic assumptions of $C, f_{\mathrm{esc}}$, and $\xi$.

In the right panel of Figure 6 the evolution of $Q$ with redshift is shown [57]. This model is broadly consistent with a wide range of observables including predictions from both LAEs, gamma ray bursts, Ly $\alpha$ forest measurements, and AGN as well as the most recent 'instantaneous reionization redshift' from Planck. Together with the $z \sim 8$ UV LF inference, it illustrates the importance of the integration limit, when predicting the ionization state of the IGM, and through that getting a handle on the unset and the end of the comic reionization. The current best estimates agree that reionization is over at redshift 6 and that a significant fraction of the IGM is still neutral at redshifts around 8 . The exact level of reionization depends on the extrapolation/integration limit of the UV LF as well as the nature of the galaxies themselves (escape fraction and ionization efficiency) and the IGM (clumping factor).

\section{Spectroscopic Confirmation of High-Redshift Galaxies}

So far the high-redshift galaxy samples described have consisted of high-redshift candidate galaxies selected based on photometry. To confirm any of these candidates as actual high redshift 

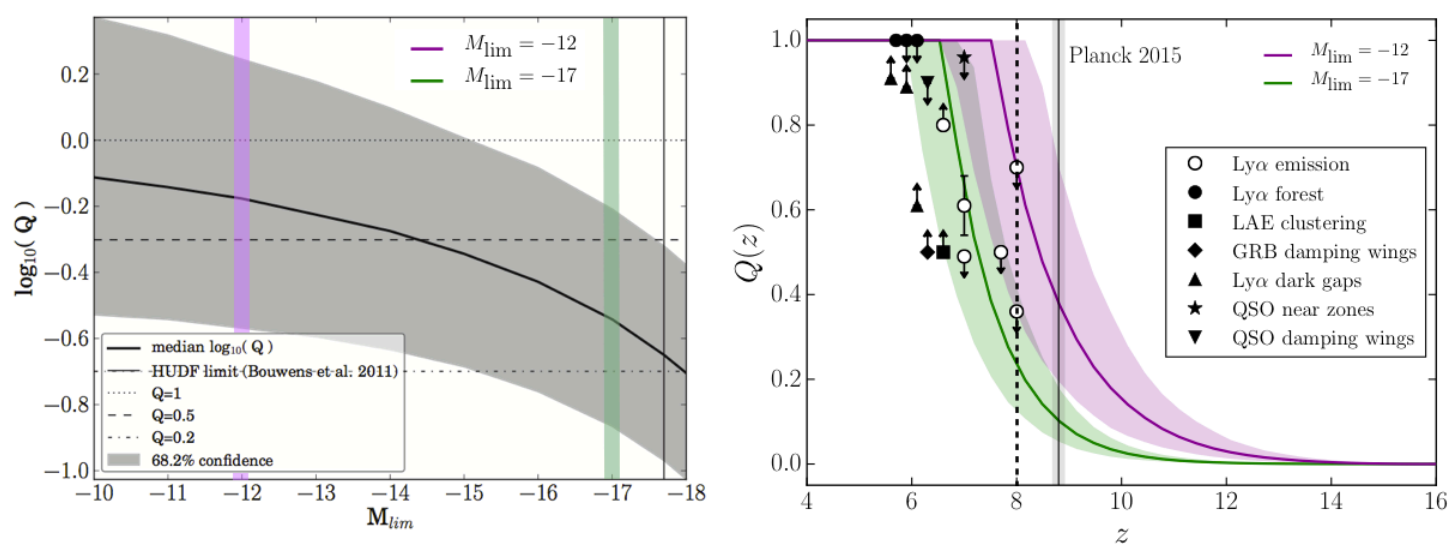

Figure 6: The left panel shows the ionized fraction, $Q$, of the intergalactic medium (IGM) as a function of the integration limit (the absolute magnitude down to which the UV LF is extrapolated) for the BoRG $z \sim 8$ LF [21]. The ionization fraction is estimated assuming a flat prior on the IGM clumping factor $C \in[1-6]$ and a photon escape fraction $f_{\text {esc }} \in[0.1-0.5]$. The horizontal dashed lines mark a 20\%, 50\% and 100\% ionized IGM. The vertical solid line shows the HUDF observational limit of -17.7 [49]. Only if objects fainter than $M_{\mathrm{UV}} \sim-15$ are included, a fully ionized IGM can be sustained at $z \sim 8$. The vertical magenta and green bands mark the integration limits of the two $Q(z)$ curves from the model linking star formation across cosmic time to the assembly of dark matter halos shown in the right panel [57]. These predictions are in good agreement with the 'instantaneous reionization redshift' from Planck (vertical solid line) and most other observations (see [57] for details) and determine the end of EoR to happen between redshift 6 and 8. The vertical dashed line, mark the location where the predictions from the BoRG $z \sim 8$ (left panel) would fall. Both panels illustrated the importance of the integration (extrapolation) limit of the UV LF when performing EoR inference.

galaxies, follow-up spectroscopy in the NIR is necessary. Confirming galaxies at high redshift is currently done by detection of the Ly $\alpha$ line, and in particular, by detection of the asymmetry of the Ly $\alpha$ line, even though other rest-frame UV lines have been proposed as alternatives [47, 48, 74]. Numerous efforts have been dedicated to detecting Ly $\alpha$ in high-redshift galaxies [23, 75, 76, 77 , $78,79,80,81,82,83,84,85,86,87,88,89,90,91,92,93]$. This has resulted in a continuously growing sample of LAEs at high redshift. In the left panel of Figure 7 two examples of such LAEs are shown. On the top, a LAE discovered independently in Keck DEIMOS spectroscopy and HST grism spectroscopy at $z=6.76[37,93]$ is shown. The bottom left panel shows the current redshift record holder confirmed with Keck MOSFIRE at $z=8.68$ [91].

Despite the many efforts, so far only a handful of sources have been confirmed above redshift $7[43,86,89,91,97,100,101,102]$. This, somewhat limited, success is partially due to the challenges with doing ground-based spectroscopic follow-up of LBG samples in the NIR. First of all, the pre-selection is challenging and in many cases contaminated by low-redshift sources, as discussed above. Secondly, the NIR sky is extremely bright, full of sky-lines and has atmospheric absorption making NIR spectroscopy challenging and expensive, as long exposure times are needed to detect the $\operatorname{Ly} \alpha$ emission in the relatively faint LBGs. This has the consequence that only the most promising and brightest candidates are currently being followed up, potentially biasing the parent 

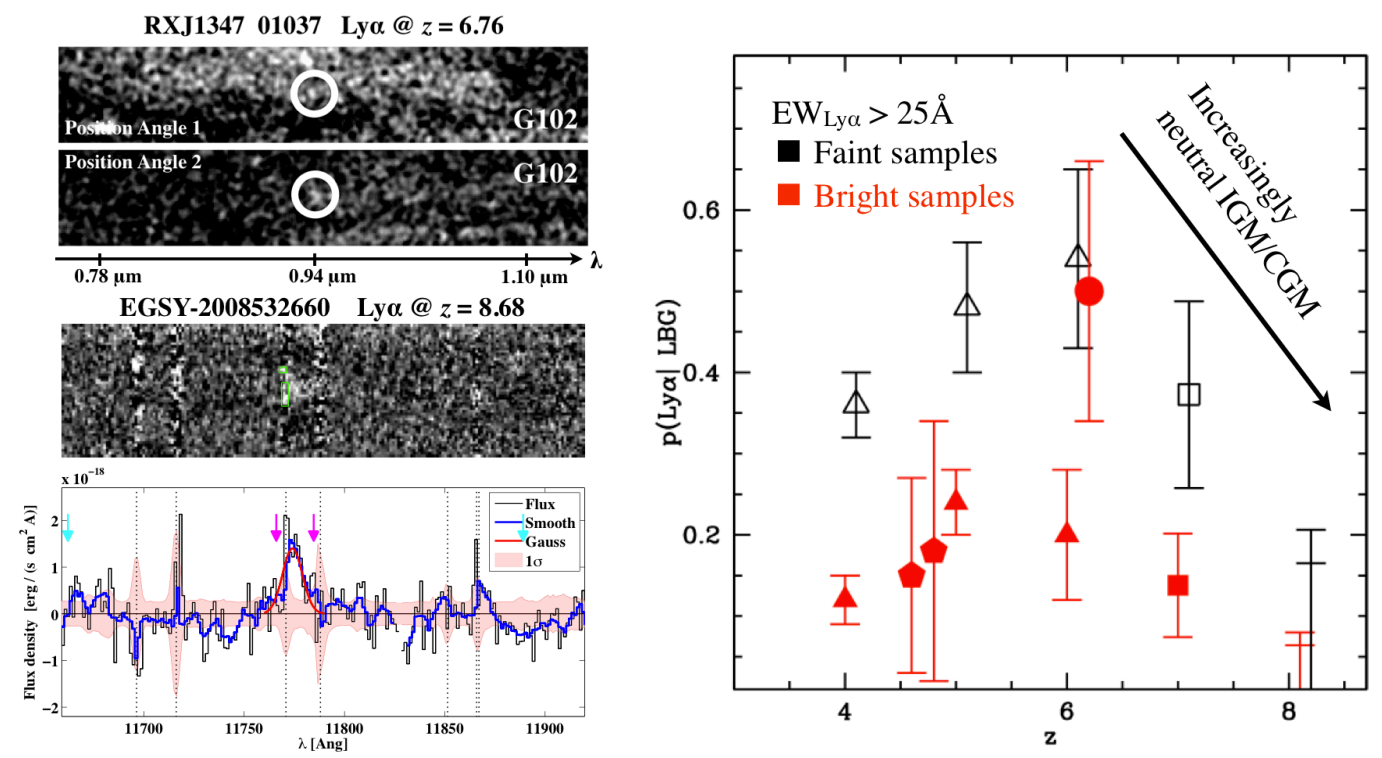

Figure 7: Two examples of LAEs confirmed in LBG samples (left). The top panel shows the GLASS [94, 95] spectra of a LAE at $z=6.76$ independently confirmed with Keck DEIMOS spectroscopy [37, 93]. The bottom panel shows the Keck MOSFIRE spectrum of the current $z=8.68$ redshift record holder for galaxies [91]. Only a handful of such LAEs have been confirmed at $z>7$. The right panel shows the evolution of the fraction of LAE emitters (Ly $\alpha$ equivalent width $>25 \AA$ ) in bright (red points, $M_{\mathrm{UV}}<$ -20.25) and faint (black points, $M_{\mathrm{UV}}>-20.25$ ) galaxies adapted from [83]. Triangles [96, 97], pentagons [98], circle [99], and squares [82] show data from spectroscopic follow-up studies. The upper limits at $z \sim 8$ [83] confirm the strong decrease of the fraction of LAEs at high redshifts. This is indicative of an increasingly neutral IGM/CGM compared to at redshift 6, i.e., a strong increase in the Ly $\alpha$ optical depth from redshift 6 to 8 .

sample of the discovered LAEs. Taking this into consideration, a deep and complete spectroscopic survey from space would be the ideal approach to detect Ly $\alpha$ at high redshift. Previously, the large amount of imaging from HST (e.g., CLASH, HFF, CANDELS, BoRG) has been explored almost exclusively to design follow-up campaigns for ground based spectroscopic facilities (e.g., Keck and VLT). However, the synergy between the HST imaging campaigns and the available NIR grisms on $H S T$ has recently been exploited by the 3D-HST [103, 104] and GLASS [93, 94, 95] collaborations. In particular, GLASS, an HST NIR grism spectroscopy survey of 10 massive galaxy clusters, is taking full advantage of the synergy between deep photometry and complete, deep, atmosphericabsorption-free, and sky-line-free spectroscopy from space over a continuous wavelength coverage of roughly $0.8-1.7 \mu \mathrm{m}$. GLASS recently presented the first results from a search for LAEs in the first six clusters [93], from which the $z=6.76 \mathrm{LAE}$ in Figure 7 is taken.

The presence of $\operatorname{Ly} \alpha$ emission in samples of LBGs can be used as a strong indicator of the state of the reionization of the IGM. The amount of Ly $\alpha$ emission is strongly dependent on the opacity of neutral hydrogen in the IGM. If the IGM surrounding the galaxy from which the Ly $\alpha$ emission escapes is highly neutral the optical depth will be large preventing the Ly $\alpha$ radiation from being observed. A decrease in the fraction of LAEs in samples of LBGs, might therefore be a direct probe of the neutral fraction of the IGM or a probe of changes in the circumgalactic 
medium (CGM). It has been shown by several studies [78, 79, 80, 82, 83, 85, 87] that the fraction of LAEs in samples of LBGs significantly decreases beyond a redshift of 6 . The right panel of Figure 7 shows the results from inferring the probability of observing Ly $\alpha$ in bright and faint samples of LBGs [83]. This inference is based on samples of LAEs and LBGs from the literature, and follow-up spectroscopy with Keck MOSFIRE of the BoRG galaxies used to determine the UV LF at $z \sim 8$ shown in Figure 3. It is clear that the fraction of LAEs is significantly decreasing from redshift 6 to 8, which is caused by fewer Ly $\alpha$ photons escaping the presumably increasingly neutral IGM/CGM. These studies therefore indicate that reionization is far from completed at redshift 8 , in good agreement with the results from the UV LFs described in Section 4.

\section{Summary}

Over the last few decades, high-redshift galaxies have become the main focus for gaining insight into the early Universe and the EoR at $z>6$. The advancement of NIR imaging and spectroscopic capabilities, from both space and the ground, have aided the search for high-redshift galaxies. In particular the installment of WFC 3 on HST has been a corner stone in the tremendous advancement of the field. Galaxies at $z>6$ are mainly selected through the Lyman break (dropout) technique or estimates of their photometric redshift. Both of these selection methods rely on multi-band photometry in the NIR and are currently returning thousands of EoR galaxy candidates. Through the rest-frame UV LF, the increasingly larger samples of high-redshift galaxies have helped determine the evolution of the IGM at the EoR. Studies have shown that the Universe was significantly ionized at $z>8$. They also show that the cosmic SFRD is declining from the peak of star formation at redshift 2-3 all the way down to $z>8$. A potential steepening at redshifts above 8 has been inferred from the current data. This steepening, which is based on small samples of objects, is in good agreement with expectations from theoretical models. With the continued improvement of selection techniques and sample sizes, the SFRD evolution will eventually have discriminating power between different theoretical models of galaxy evolution and formation in the early Universe. Current efforts to spectroscopically confirm the photometrically selected highredshift galaxy samples have only been successful in detecting Ly $\alpha$ in a handful of objects at $z>7$. This lack of LAEs at the EoR directly probes the content of neutral hydrogen in the IGM (CGM). In agreement with estimates of the ionized fraction of hydrogen in the IGM from UV LF studies, spectroscopic follow-up campaigns show that the Universe was significantly neutral at redshift 8 .

\section{The High- $z$ Future is Bright!}

Even though we have recently seen great advancement in the knowledge about the EoR, there are still questions about the current observational frontier, that we do not know how to answer. For instance, we do currently not know if LAEs exist (are observable) at $z>8.7$. Due to the small sample sizes at redshifts above 8 , we do also not know if the potential steepening of the SFRD poses any issues when compared to galaxy evolution models. Despite the preferred explanation of the drop in the fraction of LAEs being from an increased neutral IGM, there is still the possibility that the drop in LAEs is due to changes in the galaxies themselves. As these changes have to happen on time scales of less than $\sim 0.3 \mathrm{Gyr}$ (time from redshift 8 to 6 ) they pose new challenges to 
galaxy evolution models in order to explain the observed lack of LAEs at redshift 8. Many of these questions are already being studied, but will also be the focus of the bright future of high-redshift astronomy.

Currently and in the very near future a wide range of surveys will continue to study the EoR and address the many aspects of galaxies at $z>6$ which are still unknown. Among others, these surveys include HST imaging surveys (BoRG $z \sim 9-10$ [HST-GO-13767], the Hubble Frontier Fields [HST GO/DD 13495, 13496, 13498, 13504, 14037, and 14038] and RELICS [HST-GO14096]), HST grism spectroscopy surveys (GLASS [HST-GO-13459] and CLEAR [HST-GO14227]), and Spitzer Space Telescope imaging surveys (sBoRG [SST-GO-12058] and sRELICS [SST-GO-12005]). In a couple of years the James Webb Space Telescope (JWST ${ }^{2}$ ) will be launched with near- and mid-infrared cameras (NIRCam, NIRISS, MIRI) and slitless grism, multi-object and integral field unit spectrographs (NIRSpec, NIRCAM, NIRISS, MIRI), which will far surpass the sensitivity of any IR capabilities currently available. In the more distant future WFIRST ${ }^{3}$ and the 30m-class telescope era with the GMT ${ }^{4}$, the TMT ${ }^{5}$, and the E-ELT ${ }^{6}$ will present a whole new set of capabilities not only applicable to the high-redshift Universe, but all areas of astrophysics.

Hence, the continued exploration of the EoR and the planned future facilities make the high- $z$ future bright and will keep improving our knowledge about galaxy formation and evolution in the infant Universe.

\section{Acknowledgements}

First and foremost, I would like to thank the Frank N. Bash Symposium 2015 organizing committee for the kind invitation to a very stimulating meeting. Furthermore, I would like to thank the GLASS (http://glass.astro.ucla.edu) and BoRG (http://borg.physics.ucsb.edu) collaborations, and acknowledge the financial support for GLASS (HST-GO-13459), BoRG (HST-GO-11700, 12572, and 12905) and the Refsdal follow-up program (HST-GO/DD-14041) provided by NASA through grants from the Space Telescope Science Institute, which is operated by the Association of Universities for Research in Astronomy, Inc., under NASA contract NAS 5-26555. Content of this proceeding and the referenced publications is based on observations made with the NASA/ESA Hubble Space Telescope, obtained at STScI.

\footnotetext{
${ }^{2}$ http://www.stsci.edu/jwst/

${ }^{3}$ http://wfirst.gsfc.nasa.gov

${ }^{4}$ http://www.gmto.org

${ }^{5}$ http://www.tmt.org

${ }^{6} \mathrm{https}: / /$ www.eso.org/sci/facilities/eelt/
} 


\section{References}

[1] A. Loeb Scientific American 295 (Nov, 2006) 46.

[2] R. S. Ellis First Light in the Universe (Jan, 2008) 259 (c) 2008: Springer-Verlag Berlin Heidelberg.

[3] J. S. Dunlop "The First Galaxies - Theoretical Predictions and Observational Clues", 2012 by Springer, eds. V. Bromm, B. Mobasher, T. Wiklind 396 (Jan, 2013) 223.

[4] M. Dijkstra Publications of the Astronomical Society of Australia 31 (Oct, 2014) e040.

[5] C. L. Carilli and F. Walter Annual Review of Astronomy and Astrophysics 51 (Aug, 2013) 105.

[6] P. Madau and F. Haardt ApJ Letters 813 (Nov, 2015) L8.

[7] E. Giallongo, A. Grazian, F. Fiore, et al., A\&A 578 (Jun, 2015) A83.

[8] R. J. Bouwens, G. D. Illingworth, P. A. Oesch, et al., ApJ 811 (Mar, 2015) 140.

[9] B. E. Robertson, R. S. Ellis, S. R. Furlanetto, et al., ApJ Letters 802 (Apr, 2015) L19.

[10] R. L. Barone-Nugent, J. S. B. Wyithe, M. Trenti, et al., MNRAS 450 (Jun, 2015) 1224.

[11] A. Dressler, A. Henry, C. L. Martin, et al., ApJ 806 (Jun, 2015) 19.

[12] P. A. Oesch, R. J. Bouwens, C. M. Carollo, et al., ApJ Letters 709 (Jan, 2010) L21.

[13] E. Curtis-Lake, R. J. McLure, J. S. Dunlop, et al., MNRAS 457 (Sep, 2016) 440.

[14] B. W. Holwerda, R. Bouwens, P. Oesch, et al., ApJ 808 (Jul, 2015) 6.

[15] S. L. Finkelstein, R. E. Ryan, C. Papovich, et al., ApJ 810 (Sep, 2015$) 71$.

[16] R. J. Bouwens, G. D. Illingworth, P. A. Oesch, et al., ApJ 803 (Apr, 2015) 34.

[17] P. A. Oesch, R. J. Bouwens, G. D. Illingworth, et al., ApJ 808 (Jul, 2015) 104.

[18] R. Smit, R. J. Bouwens, I. Labbé, et al., eprint arXiv 1511 (Nov, 2015) 8808.

[19] S. Lorenzoni, A. J. Bunker, S. M. Wilkins, et al., MNRAS 429 (Feb, 2013) 150.

[20] R. J. Bouwens, G. D. Illingworth, P. A. Oesch, et al., ApJ Letters 709 (Feb, 2010) L133.

[21] K. B. Schmidt, T. Treu, M. Trenti, et al., ApJ 786 (May, 2014) 57.

[22] X. Fan New Astronomy Reviews 50 (Nov, 2006) 665.

[23] E. Vanzella, M. Giavalisco, M. Dickinson, et al., ApJ 695 (Apr, 2009) 1163.

[24] D. J. Mortlock, S. J. Warren, B. P. Venemans, et al., Nature 474 (Jun, 2011) 616.

[25] S. Wilkins and E. Stanway Astronomy \& Geophysics 56 (3) (Jun, 2015).

[26] C. C. Steidel, M. Giavalisco, M. Pettini, et al., AJ Letters v.462 462 (May, 1996) L17.

[27] C. C. Steidel, K. L. Adelberger, A. E. Shapley, et al., ApJ 532 (Mar, 2000) 170.

[28] P. Madau, H. C. Ferguson, M. E. Dickinson, et al., MNRAS 283 (Dec, 1996) 1388.

[29] S. Lorenzoni, A. J. Bunker, S. M. Wilkins, et al., MNRAS 414 (Jun, 2011) 1455.

[30] D. Coe, A. Zitrin, M. Carrasco, et al., ApJ 762 (Jan, 2013) 32.

[31] S. M. Wilkins, E. R. Stanway, and M. N. Bremer MNRAS 439 (Mar, 2014) 1038.

[32] S. L. Finkelstein eprint arXiv 1511 (Nov, 2015) 5558.

[33] E. R. Stanway, A. J. Bunker, and R. G. McMahon MNRAS 342 (Jun, 2003) 439.

[34] S. Malhotra, J. E. Rhoads, N. Pirzkal, et al., ApJ 626 (Jun, 2005) 666.

[35] R. J. McLure, J. S. Dunlop, R. A. A. Bowler, et al., MNRAS 432 (Jul, 2013) 2696.

[36] D. Coe, L. Bradley, and A. Zitrin ApJ 800 (Feb, 2015) 84.

[37] K.-H. Huang, M. Bradač, B. C. Lemaux, et al., ApJ 817 (Jan, 2016) 11.

[38] J. Walcher, B. Groves, T. Budavári, et al., Astrophysics and Space Science 331 (Jan, 2011) 1.

[39] R. J. McLure, J. S. Dunlop, L. de Ravel, et al., MNRAS 418 (Dec, 2011) 2074.

[40] M. A. Schenker, B. E. Robertson, R. S. Ellis, et al., ApJ 768 (May, 2013) 196. 
[41] R. Smit, R. J. Bouwens, I. Labbé, et al., ApJ 784 (Mar, 2014) 58.

[42] R. Smit, R. J. Bouwens, M. Franx, et al., ApJ 801 (Mar, 2015) 122.

[43] G. W. Roberts-Borsani, R. J. Bouwens, P. A. Oesch, et al., eprint arXiv 1506 (Jun, 2015) 854.

[44] M. Ajiki, B. Mobasher, Y. Taniguchi, et al., ApJ 638 (Feb, 2006) 596.

[45] T. Shibuya, N. Kashikawa, K. Ota, et al., ApJ 752 (Jun, 2012) 114.

[46] J. Matthee, D. Sobral, S. Santos, et al., MNRAS 451 (Feb, 2015) 400.

[47] D. P. Stark, G. Walth, S. Charlot, et al., MNRAS 454 (Dec, 2015) 1393.

[48] D. P. Stark, J. Richard, S. Charlot, et al., MNRAS 450 (Jun, 2015) 1846.

[49] R. J. Bouwens, G. D. Illingworth, P. A. Oesch, et al., ApJ 737 (Aug, 2011) 90.

[50] P. Schechter AJ 203 (Jan, 1976) 297.

[51] M. Trenti, L. D. Bradley, M. Stiavelli, et al., ApJ Letters 727 (Feb, 2011) L39.

[52] L. D. Bradley, M. Trenti, P. A. Oesch, et al., ApJ 760 (Dec, 2012) 108.

[53] R. Bowler Astronomy \& Geophysics 56 (3) (Jun, 2015).

[54] R. A. A. Bowler, J. S. Dunlop, R. J. McLure, et al., MNRAS 440 (May, 2014) 2810.

[55] R. A. A. Bowler, J. S. Dunlop, R. J. McLure, et al., MNRAS 452 (Sep, 2015) 1817.

[56] J. S. B. Wyithe, H. Yan, R. A. Windhorst, et al., Nature 469 (Jan, 2011) 181.

[57] C. A. Mason, M. Trenti, and T. Treu ApJ 813 (Nov, 2015) 21.

[58] C. A. Mason, T. Treu, K. B. Schmidt, et al., ApJ 805 (May, 2015) 79.

[59] A. Fialkov and A. Loeb ApJ 806 (Jun, 2015) 256.

[60] P. Madau, L. Pozzetti, and M. Dickinson ApJ 498 (May, 1998) 106.

[61] R. C. Kennicutt Annu. Rev. Astro. Astrophys. 36 (Jan, 1998) 189.

[62] P. Madau and M. Dickinson Annual Review of Astronomy and Astrophysics 52 (Aug, 2014) 415.

[63] P. A. Oesch, R. J. Bouwens, G. D. Illingworth, et al., ApJ 773 (Aug, 2013) 75.

[64] P. A. Oesch, R. J. Bouwens, G. D. Illingworth, et al., ApJ 786 (May, 2014) 108.

[65] D. J. McLeod, R. J. McLure, J. S. Dunlop, et al., MNRAS 450 (Jul, 2015) 3032.

[66] J. M. Shull, A. Harness, M. Trenti, et al., ApJ 747 (Mar, 2012) 100.

[67] B. E. Robertson, S. R. Furlanetto, E. Schneider, et al., ApJ 768 (May, 2013) 71.

[68] J. S. Dunlop, R. J. McLure, B. E. Robertson, et al., MNRAS 420 (Feb, 2012) 901.

[69] R. J. Bouwens, R. Smit, I. Labbe, et al., eprint arXiv 1511 (Nov, 2015) 8504.

[70] A. A. Kaurov and N. Y. Gnedin ApJ 787 (Jun, 2014) 146.

[71] K. Duncan and C. J. Conselice MNRAS 451 (Aug, 2015) 2030.

[72] S. M. Wilkins, Y. Feng, T. D. Matteo, et al., MNRAS 458 (Jan, 2016) L6.

[73] S. M. Wilkins, R. J. Bouwens, P. A. Oesch, et al., MNRAS 455 (Jan, 2016) 659.

[74] D. P. Stark, J. Richard, B. Siana, et al., MNRAS 445 (Dec, 2014) 3200.

[75] E. Vanzella, A. Fontana, L. Pentericci, et al., A\&A 569 (Sep, 2014) A78.

[76] E. Vanzella, A. Fontana, A. Zitrin, et al., ApJ Letters 783 (Mar, 2014) L12.

[77] L. Pentericci, A. Fontana, E. Vanzella, et al., ApJ 743 (Dec, 2011) 132.

[78] L. Pentericci, E. Vanzella, A. Fontana, et al., ApJ 793 (Oct, 2014) 113.

[79] J. Caruana, A. J. Bunker, S. M. Wilkins, et al., MNRAS 427 (Dec, 2012) 3055.

[80] J. Caruana, A. J. Bunker, S. M. Wilkins, et al., MNRAS 443 (Oct, 2014) 2831.

[81] M. Bradač, E. Vanzella, N. Hall, et al., ApJ Letters 755 (Aug, 2012) L7.

[82] T. Treu, M. Trenti, M. Stiavelli, et al., ApJ 747 (Mar, 2012) 27. 
[83] T. Treu, K. B. Schmidt, M. Trenti, et al., ApJ Letters 775 (Sep, 2013) L29.

[84] I. Balestra, E. Vanzella, P. Rosati, et al., A\&A 559 (Nov, 2013) L9.

[85] V. Tilvi, C. Papovich, S. L. Finkelstein, et al., ApJ 794 (Oct, 2014) 5.

[86] M. A. Schenker, R. S. Ellis, N. P. Konidaris, et al., ApJ 795 (Nov, 2014) 20.

[87] A. L. Faisst, P. Capak, C. M. Carollo, et al., ApJ 788 (Jun, 2014) 87.

[88] W. Karman, K. I. Caputi, C. Grillo, et al., A\&A 574 (Feb, 2015) A11.

[89] P. A. Oesch, P. G. van Dokkum, G. D. Illingworth, et al., ApJ Letters 804 (May, 2015) L30.

[90] D. Watson, L. Christensen, K. K. Knudsen, et al., Nature 519 (Mar, 2015) 327.

[91] A. Zitrin, I. Labbé, S. Belli, et al., ApJ Letters 810 (Sep, 2015) L12.

[92] A. Hoag, M. Bradač, K. H. Huang, et al., ApJ 813 (Nov, 2015) 37.

[93] K. B. Schmidt, T. Treu, M. Bradač, et al., ApJ 818 (Feb, 2016) 38.

[94] K. B. Schmidt, T. Treu, G. B. Brammer, et al., ApJ Letters 782 (Feb, 2014) L36.

[95] T. Treu, K. B. Schmidt, G. B. Brammer, et al., ApJ 812 (Sep, 2015) 114.

[96] D. P. Stark, R. S. Ellis, and M. Ouchi ApJ Letters 728 (Feb, 2011) L2.

[97] M. A. Schenker, D. P. Stark, R. S. Ellis, et al., ApJ 744 (Jan, 2012) 179.

[98] R. P. Mallery, B. Mobasher, P. Capak, et al., ApJ 760 (Dec, 2012) 128.

[99] E. Curtis-Lake, R. J. McLure, H. J. Pearce, et al., MNRAS 422 (May, 2012) 1425.

[100] E. Vanzella, L. Pentericci, A. Fontana, et al., ApJ Letters 730 (Apr, 2011) L35.

[101] Y. Ono, M. Ouchi, B. Mobasher, et al., ApJ 744 (Jan, 2012) 83.

[102] S. L. Finkelstein, C. Papovich, M. Dickinson, et al., Nature 502 (Oct, 2013) 524.

[103] I. G. Momcheva, G. B. Brammer, P. G. van Dokkum, et al., eprint arXiv 1510 (Oct, 2015) 2106.

[104] G. B. Brammer, P. G. van Dokkum, M. Franx, et al., ApJ Supplement 200 (Jun, 2012) 13. 